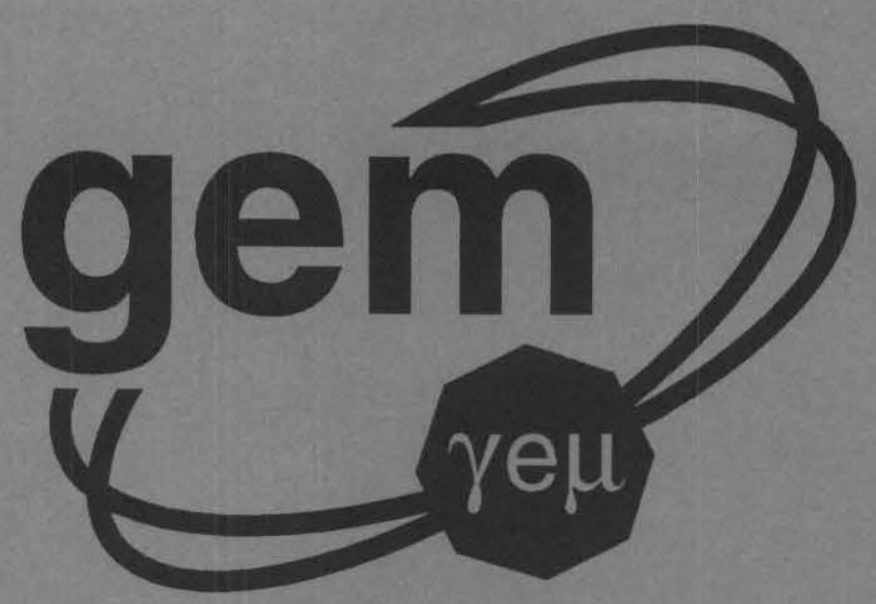

\title{
Module of Electromagnetic Secondary Emission Flight Type Calorimeter
}

G. S. Bitsadze, M. I. Chernetsov, I. V. Manuilov, A. M. Medvedkov V. I. Rykalin, G. V. Zholobov

Institute for High Energy Physics, Protvino, Russian Federation

Yu. V. Khrenov, V. T. Tolmachev

Joint Institute of Nuclear Research, Dubna, Russian Federation

M. Kobayashi

KEK, Tsukuba-Shi, Japan

June 29, 1993

\section{Abstract;}

The prototype of the module of Electromagnetic Secondary Emission Flight Type Calorimeter is discribed. The design, first experimental results, obtained in particle beams, and Monte Carlo calculations, are presented. The energy resolution of the calorimeter for $26 \mathrm{Ge} \mathrm{V}$ electrons is $\sigma_{E} / \mathrm{E} \approx 23 \%$. 


\title{
Module of Electromagnetic Secondary Emission Flight Type Calorimeter
}

\author{
G.S.Bitsadze ${ }^{1)}$, M.I.Chernetsov ${ }^{1)}$, Yu.V.Khrenov'), M.Kobayashi' ${ }^{3)}$, \\ I.V.Manuilov' ${ }^{1)}$,A.M.Medvedkov'), V.I.Rykalin ${ }^{1)}$, V.T.Tolmachev ${ }^{2)}$, \\ G.V.Zholobov ${ }^{1)}$
}

June 29, 1993

\begin{abstract}
The prototype of the module of Electromagnetic Secondary Emission Flight Type Calorimeter is discribed. The design, first experimental results, obtained in particle beams, and Monte Carlo calculations, are presented. The energy resolution of the calorimeter for $26 \mathrm{GeV}$ electrons is $\sigma_{E} / \mathrm{E} \approx 23 \%$.
\end{abstract}

For planned collider detectors [1] the required characteristics of forward calorimeters are similar (see table 1). As a rule, this is a hadron calorimeter with low energy resolution, but having a radiation hardness exceeding $100 \mathrm{Mrad} / \mathrm{yr}$. Presently new approaches to building such calorimeters are being studied or new types of detectors are being tested. The characteristics of the radiation hardness of some types of calorimeter candidates are presented in table 2.

Ionization calorimeters can be used as forward calorimeters if resolution of the problem of it front-end electronics can moved away from the detector without distortion of general characteristics [2].

In the last years heavy monocrystals $\left(\mathrm{CeF}_{3}, \mathrm{GSO}\right)$ have been synthesized, with transparency changes at the level of $1 \%$ for a total dose of $100 \mathrm{Mrad}$ [3] ( $\gamma$ source:

\footnotetext{
${ }^{1}$ Institute for High Energy Physics, Protvino, Russian Federationon

3Joint Institute of Nuclear Research, Dubna, Russian Federation

${ }^{3}$ KEK, Tsukuba-Shi, Japan
} 
${ }^{60} \mathrm{Co}$ ). However, observed percent variations of the transparency per one radaiation length $X_{0}$ correspond to some tens of percent for a full-scale electromagnetic calorimeter of (20-25) $X_{0}$ or for hadron calorimeter with real longitudinal segmentation (5-10 sections). Apart from this, the question of how to make a photomultiplier (PM), having the radiation hardness of tens or hundreds of Mrad is not solved yet. One also should take into account the cost of such monocrystals. For example, electromagnetic calorimeters based on GSO monocrystals with thickness of $20 X_{0}$, would cost at least $5 \times 10^{6} \mathrm{US} \$ / \mathrm{m}^{2}$, according to our estimations.

A priori, secondary emission of metals and semiconductors should be little affected by radiation. Calculations of the radiation dose absorbed by the surfaces of strong-current photomultiplier dynodes from the flux of secondary electrons during their guaranteed life-time gives a value which exceeds by many orders of magnitude the required one. Therefore, utilization of secondary emission for obtaining signals from showers in calorimeters naturally attracts the detector developers $[4,5]$. To verify the assumption of a potentially high radiation hardness of the secondary emission emitters, authors of the present article have measured the radiation hardness of the dynode system of the PM FEU-110 with $\mathrm{CuAlMg}$-alloy dynodes. Before exposure to $\gamma$ quanta from ${ }^{60} \mathrm{Co}$, the photocurrent in the photocathode circuit and amplitudes of the output signals from the PM were measured. After the exposure the values of the photocathode currents and amplitudes of the signals from PM were measured again. With an integred radiation dose of $100 \mathrm{Mrad}$, the photocurrent was reduced by factor of two, which could be due to a noticeable darkening of the photocathode window. The amplification of the PM, which is easily determined from the consideration of the photocurrent variation, changed (increased) by $5 \%$, with a comparable measurment accuracy.

The application of microchannel plates (MCP) in sampling-calorimeters [4] actually solves the problem, with an accuracy of contradictory data about the radiation hardness of MCP and the possibility of practical implementation of such a detector.

For detection of the electromagnetic and hadronic showers authors of the paper [5] proposed the use of secondary emission electrons, knocked out by the shower particles from the surfaces of the absorber layers. Those electrons are transported through the stacks of absorbers and amplified further by a secondary electron multiplier (SEM). The possibility of building such a detector was tested with the help of a three-layer prototype, irradiated by electrons obtained from a special emitter.

In this article the prototype of a module of an electromagnetic secondary emission flight type calorimeter (SEFCAL) is described. The Monte Carlo calculations and the first experimental results of the module study in particle beams are presented. 
The authors consider the development of the module as a first step toward the hadron calorimeter design, though, the electromagnetic SEFCAL design is of independent interest.

\section{Calorimeter Module Design.}

The module of the electromagnetic calorimeter is presented in fig.1. It consists of 50 layers of the absorber. Each absorber layer is a set of $100 \times 6 \times 1.5 \mathrm{~mm}^{3}$ lead plates fixed on a brass frame at an angle of $45^{\circ}$. The front wall of each layer of the absorber has a $0.5 \times 0.5 \mathrm{~mm}^{2}$ fine-grain metal grid to shield the surfaces of the lead plates from interlayer electric field. The absorber layers are hold by 4 sets of rods and bushings (see fig.1). The distance between the layers is $2 \mathrm{~mm}$. An equal potential difference is applied to the neighbouring layers of the absorber with the help of a uniform divider having a base resistance of $3 \mathrm{MOhm}$. The design of the module is actually similar to that of shutter-type dynode systems ("venetian blind" system), e. g. of PM FEU-110.

With a chosen thickness of the lead strips of $1.5 \mathrm{~mm}$, the total downstream length of the module is $\approx 18 X_{0},\left(X_{0} \approx 25 \mathrm{~mm}\right.$ is a radiation length). The number of layers was determined by the following consideration. It is known, that the secondary emission efficiency of a metal surface penetrated by relativistic charged particles is $2-4 \%$ [6] for perpendicularly incident particles. Therefore, in order to obtain a few secondary electrons from a single charged particle (muon, hadron) passing through all layers, the number of the layers should be equal to at least 50 (100 surfaces). The results of the calculations, presented below, shows that the choice of the optimal number of layers is actually a many-parameter problem.

The front part of the module incorporates a system of two shutter dynodes from the PM FEU-110, one being the photo emitter and the other is used for multiplication of secondary emission. When this system is illuminated by $10 \mathrm{nsec}$ light pulses from an ultraviolet laser, clusters of secondary electrons come from the first layer of the module. They are used as standard charged signals for checking and module calibration.

At the back of the module was SEM, which is a PM FEU-49B with photocathode window removed. 


\section{Vacuum System.}

The calorimeter module was placed into a stainless steel container of cylindrical shape, $320 \mathrm{~mm}$ in diameter and $900 \mathrm{~mm}$ long. In the front part of the container there is a quartz glass window to inject a laser ray (fig.2).

When manufacturing the vacuum system and module prototype, we took into account the known fact, that secondary emission multipliers are working well at the pressures below $10^{-5}$ Torr. The required dynamic vacuum was produced by a system of forevacuum and oil-vapour pumps without outgassing of the container and module. In the vacuum system an oil-vapour pump, having an air pumping rate of at least $200 \mathrm{l} / \mathrm{sec}$, was used. The container was connected to the high-vacuum chamber by a tube of $100 \mathrm{~mm}$ in diameter and $200 \mathrm{~mm}$ long. It should be noted, that the laser signals at the SEM output appeared when a vacuum of $\leq 10^{-4}$ Torr was attained, in 40-60 minutes after the beginning of pumping. During the experimental study the operational pressure of $(2-4) \times 10^{-6}$ Torr was attainable after $2-3$ hours.

\section{Measurement of the Layer Multiplication Coefficient.}

One of the basic parameters of this calorimeter is a coefficient of secondary electron multiplication on the absorber layers. It should be noted, that this coefficient must be the same for all layers and close to unity, because otherwise the statistical weight of the signals coming from different layers will be different, leading to a deterioration of the resolution. The coefficient was measured for an assembly consisting of 5 layers (see fig.3). The photocathode-secondary emitter system, similar to the one shown in fig.1, was installed in the front part of the assembly. It was illuminated by a ultraviolet lamp. The obtained photocurrent $I_{0}$ was collected by a copper plate anode, placed immediately after it (position is shown by the dashed line in fig.3). Then the anode was placed after 5 layers and the current $I_{5}$ was measured. The coefficient of layer multiplication $k_{\text {mult }}$ was found from the ratio of $I_{0}$ and $I_{5}$. The measured $k_{\text {mult }}$ dependence upon the intercascade voltage $U_{c}$ is shown in fig.4. It is seen, that for the given design and material of the layers, the multiplication coefficient $k_{\text {mult }}$ varies from 0.85 to 1.2 . 


\section{Study of the Secondary Emission Flight Type Calorimeter Module Characteristics.}

The characteristics of the calorimeter module were studied with the help of an ultraviolet laser and in particle beams.

Excitation of the calorimeter module by a pulsed laser (see fig.2) allowed us to make routine measurements of its reliability, and what is more important, to determine the nature of signal fluctuations and the linearity of the whole system versus the charge of secondary electron clusters injected into the module. The cluster charge was varied by laser beam attenuation by Plexiglas plates. The relative intensity of the laser light pulses was measured independently with the help of a sun blind vacuum photoelement, detecting reflected light from the optical input window. For example, fig. 5 shows the amplitude spectra of signals at the module output, corresponding to the unattenuated laser beam (the right-hand spectrum) and the laser beam after passing through a $18 \mathrm{~mm}$ Plexiglas plate (the left-hand spectrum). Figure 6 presents the dependence of $(2 \tau / \mathrm{A})^{2}$ on $1 / \mathrm{A}$, where $2 \tau$ is FWHM and $\mathrm{A}$ is the mean amplitude of the amplitude spectrum. The dependence is linear and crosses 0 , showing the linearity of the detecting system (including the electronics) and to the Gaussian nature of fluctuations, which is related only with the mean number $N_{e}$ of secondary emission electrons at the SEM input. Indeed, for a Gaussian distribution, $2 \tau \sim \sqrt{N_{e}}$, and from the linearity, $N_{e} \sim \mathrm{A}$.

The characteristics of the calorimeter, exposed to particles, were studied with $26 \mathrm{GeV} / \mathrm{c}$ electron and muon beams. The beam momentum spread was $\Delta \mathrm{p} / \mathrm{p} \approx 1 \%$. The output signals from the calorimeter module were sent to 12-bit ADC[7]. The amplitude spectra for $26 \mathrm{GeV}$ electrons, measured for various values of the intercascade voltage $U_{c}$, are shown in fig.7a-7d. From comparison of the spectra it is seen, that with an increase of $U_{c}$, which results in a growth of the multiplication coefficient $k_{\text {mult }}$, the resolution deteriorates. The best resolution of the calorimeter module, obtained at $k_{\text {mult }}=1.15$ for $26 \mathrm{GeV}$ electrons, is $\sigma_{E} / E \approx 23 \%$.

Fig. 8 shows the amplitude spectrum for muons passing through all calorimeter layers. The efficiency of the muon detection is about $50 \%$. 


\section{Monte Carlo Simulation. Comparison with the Experiment.}

The detailed simulation of the physical processes, determining the physical result under study, simplifies the search for the optimal parameters and operational modes of the calorimeter. The model we used is described in brief below.

The electromagnetic shower for the specified geometry of the calorimeter was simulated with the help of the GEANT code [8]. The development of the shower was traced to the minimum kinetic energy allowed in the code, $10 \mathrm{keV}$. During the simulation of secondary emission electrons it was assumed that every particle of the shower, when crossing the absorber surface, knocks out of it a slow secondary emission electron with a probability of $w \approx(2-4) \%[6]$. During the transportation of secondary emission electrons by electric field to the anode, they undergo collisions with the absorber plates. In each collision the number of secondary electrons varyed in accordance with Poisson distribution and is determined by the layer multiplication coefficient $k_{\text {mult }}$. Its value is close to unity and, as it was shown above, depends on the intercascade voltage. Hence, the model under consideration depends on two parameters, $k_{\text {mult }}$ and $w$. The simulation results are presented in table 3 . In the computations the angular dependence of $w$ was not taken into account and its value for perpendicularly incident particles was used. Besides, the exact value of $w$ is not known for the lead surface having a natural oxide film, which should increase w, similar to the influence of a CsI film [9]. Hence, the results mainly allow one to trace the dependence of the energy resolution and other characteristics of the detector upon various parameters.

The results presented in table 3 were obtained at energies of 5 and $26 \mathrm{GeV}$ (column 2), for various values of the geometric parameters of the module i.e. the thickness of the absorber plates (column 4) and their transverse dimensions (column 9 ). The dependences of the detector characteristics on the parameters $w$ (column 3 ) and $k_{\text {mult }}$ (column 10 ) were studied. In the case of the calorimeter depth segmentation, we indicate the segmentation structure (the number of layers in each section) in column 5. The arrows under the digits indicate the directions of secondary emission electron collection in sections. For the nonsegmentation case the downstream direction was standard. Columns 6-8 present the energy resolution $\sigma_{E} / E$ recalculated to $1 \mathrm{GeV}$, the number of secondary emission electrons $N_{e}$ and the energy deposition $\Delta E$ in the calorimeter, respectively. For calculations with no Poisson fluctuations, the calorimeter structure is the same, as for the previous calculations. 
Some results calculated for 5 and $26 \mathrm{GeV}$ are presented in figs.9-11, respectively. Fig.9 shows the spectrum of the total energy deposition in the calorimeter (in \% of the particle energy causing the shower), the longitudinal and transverse distributions of the shower and the measured charged spectrum (distribution $N_{e}$ ), in the absence of Poisson fluctuations of secondary emission electron number. Figs.10-11 show the charged longitudinal shower profile and the charged spectra for different values of the parameters $\mathrm{w}$ and $k_{\text {mult }}$ at 5 and $26 \mathrm{GeV}$. The titles in figs. $9 \div 11$ characterizes the energy, the thickness of the plates in $\mathrm{cm}$, the number of layers and the transverse module dimensions in $\mathrm{cm}^{2}$.

Before going over the discussion of the data of Table 3 , let us make the following two remarks:

1) To check an accuracy level of the simulation code we have done calculations for a $4 \times 4 \mathrm{~cm}^{2}$ cell of the lead - scintillator sandwich type electromagnetic calorimeter $(0.2 \mathrm{~cm}$ lead and $0.5 \mathrm{~cm}$ scintillator). The obtained results are in a good agreement with the experiment [10].

2) The calculations for a $52 \times 52 \mathrm{~cm}^{2}$ module are presented to determine the effect of the lateral leakage of the shower on the calorimeter characteristics.

From the data presented in table 3 it follows that:

1) The comparison of the energy resolution values at $5 \mathrm{GeV}$ with Poisson fluctuations of the secondary emmision electrons number $(N=6,15,19)$ and in their absence $(7,16,20)$ shows that Poisson fluctuations make the main contribution into the energy resolution, degrading it by a factor of 3 . At $26 \mathrm{GeV}$ the relative contribution from the Poisson fluctuations is less, but still remains significant (23 and 24).

2) It follows from $\mathrm{N}=1.5$ of table 3 , that the absorber plates thickness (with unchanged total calorimeter length in the units of $X_{0}$ ) does not noticeably affect the resolution, i.e. a growth of sampling fluctuations for increasing thickness is compensated by a decrease of Poisson fluctuations due to a lower number of layers. However, the value of the signal decreases for increasing plate thickness.

3) Two values of the parameter $w$ are used: 0.025 and 0.05 . The resolution improves with a growth of $\mathrm{w}(\mathrm{N}=1$ and 6,2 and 19), indicating that the value of w must be determined more accurately.

4) The results are dependent most noticeably on the parameter $k_{\text {mult }}$, which was varied from 0.9 to 1.165 . The $\mathrm{N}=6,8,9$ and $23,25-32$ of the table show, that with a $k_{\text {mult }}$ deviation from one the resolution worsens. In addition, the comparison of the charged longitudinal distributions of the showers, relevant to $k_{\text {mult }}=1$ and $k_{m u l t}=1.13$ (figs. 10 and 11 , respectively), shows that with an increase of $k_{\text {mult }}$ the charged 
longitudinal profile of the shower is distorted. This results in the energy nonlinearity of the calorimeter response (column 7 of $N=17,30$, compared with $N=15,23$ ) and in distortion of the generally accepted dependence $\sigma_{E} / E$. Indeed, the relative energy resolution values for 5 and $26 \mathrm{GeV}$, recalculated to $1 \mathrm{GeV}$ according to the usual dependence $\sigma_{E} / E \sim 1 / \sqrt{E}$, are sharply different (coloumn 6 of the specified $\mathrm{N}$ ). Hence, the value of $k_{\text {mult }}$ should be close to unity.

5) The energy resolution of the calorimeter at $5 \mathrm{GeV}$ for $1.5 \mathrm{~mm}$ thick absorber plates is $33.5 \%(w=0.05)$ and $46.5 \%(w=0.025)(\mathrm{N}=1$ and 6 , respectively). The resolution is somewhat higher for $3 \mathrm{~mm}$ thick absorber plates, $31.3 \%$ and $41.8 \%$ (N $=2$ and 19, respectively). The last version is preferable, because it makes the design of the calorimeter more simple. The same is true for $26 \mathrm{GeV}$.

6) The depth segmentation of the calorimeter allows one to decrease the contribution of Poisson fluctuations to the energy resolution $(N=10-13,21)$. For example, segmentation just into 4 parts makes it possible to attain the resolution close to the best without Poisson fluctuations. For $\mathrm{N}=13$ with the segmentation and charge collection as specified in column 5 , the resolution is $24.9 \%$, which is close to the maximum one, $15.2 \%(\mathrm{~N}=7)$. In addition, in the case of segmentation, even for $k_{\text {mult }}=1.165$ the calorimeter is linear in energy, its resolution is sufficiently high ( $N$ $=33,18$ ) and much more weakly dependent on $k_{\text {mult }}$ (cf. $\mathrm{N}=13,14$ and 6,9 ) with relatively small weakening of the dependence on parameter $w$ (cf. $N=2,19$ and 21,22).

7) The muon detection efficiency is strongly dependent on $k_{\text {mult }}$. It varies from $30 \%\left(k_{\text {mult }}=1\right)$ upto $80 \%\left(k_{\text {mult }}=1.165\right)$.

Fig.12 compares the calculated dependence of $\sigma_{E} / E$ on $k_{\text {mult }}$ with the experimental data, obtained in this work. It is seen, that the calculated and experimental measurements of the energy resolution value differ significantly and this cannot be explained by the uncertainty in the value of $\mathrm{w}(\mathrm{N}=30,34)$. One possible reason for this difference may be the assumption of passage of secondary electrons through the absorber layers without collisions. This changes the character of the distribution of the number of secondary electrons obtained in the calculations by decreasing its dispersion. This assumption needs to be checked carefully, because it can lead to the improvement of the energy resolution of similar calorimeters and decreases the time of flight of secondary electrons. The triangle in this figure denotes the Monte Carlo result for calorimeter segmented into 4 parts at $5 \mathrm{GeV}(\mathrm{N}=13)$, recalculated to $26 \mathrm{GeV}$. The result presented characterizes the best attainable resolution for the chosen structure. 
The authors would like to express their deep gratitude to Yu.D. Prokoshkin for the support of the present work and fruitful discussions, to V.I.Kireev, V.I.Spiryakin, Yu.P.Petukhov, A.M.Gorin, V.P.Djordjadze and V.P.Sugonyaev and also to the teams of MIS ITEP and PROZA for their assistance in measurements. 


\section{Figure Captions}

Fig.1 The schematic view of the secondary emission flight type calorimeter: 1shutter dynodes from a photoelectron multiplier, 2-glass rod, 3-glass bushing, 4-brass frame, 5-lead strip of the absorber, 6-metal grid, 7-secondary electron multiplier, 8-amplifier.

Fig.2 The scheme of studying of secondary emission flight type calorimeter.

Fig.3 The scheme of measurment of the layer multiplication coefficient $\boldsymbol{k}_{\text {mult }}$ dependence upon the intercascade voltage $U_{c}$.

Fig.4 The multiplication coefficient $k_{m u l t}$ of the layer versus the intercascade voltage $U_{c}$.

Fig.5 The amplitude spectra from the ultraviolet laser: the right-hand spectrumthe laser ray is not attenuated, the left-hand spectrum-attenuated by crossing of $18 \mathrm{~mm}$ Plexiglas.

Fig.6 The dependence of $(2 \tau / A)^{2}$ upon 1/A.

Fig.7 The experimentally measured amplitude spectra for $26 \mathrm{GeV}$ electrons for different values of the intercascade voltage $U_{c}\left(k_{\text {mult }}\right)$.

Fig.8 The experimentally measured amplitude spectrum of muons.

Fig.9 The basic calculated characteristics of the electromagnetic shower in secondary emission flight type calorimeter (SEFCAL): (a) the spectrum of the total energy deposition in the calorimeter in \% of the energy causing the particle shower; (b) the longitudinal distribution of electromagnetic shower-the relative energy deposition vs the number of layers; (c) the transverse distribution of the shower-the relative energy deposition vs the number of longitudinal strips in a layer, summed over the whole depth; (d) the measured charged spectrum $N_{e}$ in absence of Poisson fluctuations of the number of secondary emission electrons.

Fig.10 The charged longitudinal distribution of shower-the average numbers of secondary emission electrons vs the number of layers (left) and the charged spectra (right) for different values of the parameters $k_{\text {mult }}$ and $w$ at 5 $\mathrm{GeV} / \mathrm{c}$. 
Fig.11 The charged longitudinal distribution of shower-the number of emission electrons vs the number of layers (left) and the charged spectra (right) for different values of the parameters $k_{\text {mult }}$ and $w$ at $26 \mathrm{GeV} / \mathrm{c}$.

Fig.12 The comparison of the calculated dependence of $\sigma_{E} / E$ upon $k_{m u l t}$ with the experimental data. 


\section{References}

[1] Letters of Intent to the SSC Laboratory from EMPACT, ${ }^{\star}$, TEXAS and SD Collaborations, 1990.

[2] R.N.Krasnokutsky et al. Cable Connections for Detector and Preamplifier, Eliminating Radiation Damage of Electronics. Preprint IHEP 91-48, Protvino 1991.

[3] M.Kobayashi et al., Nucl. Instr. Meth. A302(1991)443; G.I.Britvich et al. Nucl. Instr. Meth. A308(1991)509; M.Kobayashi and M.Ishii, Nucl. Instr. Meth. B61(1991)491.

[4] A.A.Derevshchikov et al., On Possibility to Make a New Type of Calorimeter: Radiation Resistant and Fast. IHEP preprint 90-99, Protvino, 1990.

[5] G.S.Bitsadze, A.M.Medvedkov and V.I.Rykalin, On the Possibility of Design of Secondary Emission Flight Type Calorimeter, III WORKSHOP "PHYSICS AT UNK", 25-28 September 1990, Protvino, USSR, p.21.

[6] V.Agoritsas, Secondary Emission Chambers for Monitoring the CPS Ejected Beams, SYMPOSIUM ON BEAM INTENSITY MEASUREMENT, Daresbury,22-26 April 1968, p.117.

[7] F.Binon et al., Data Acquisition System for the GAMS-2000 Spectrometer, Preprint IHEP 88-179, Serpukhov 1988.

[8] R.Brun et al., Preprint CERN - DD/EE/84-1, 1987.

[9] H.L.Seifert et al., Increased Secondary Electron Yield from Thin CsI Coatings, LA UR -90-713, Los-Alamos, 1990.

[10] A.N.Vasiliev et al. Preprint IHEP 82-62, Serpukhov, 1982. 
Table 1: Requirements on forward calorimeters in SSC and LHC projects

\begin{tabular}{|c|c|c|c|c|}
\hline Projects & $\begin{array}{c}\text { Calorimeter } \\
\text { type }\end{array}$ & $\begin{array}{c}\text { Energy } \\
\text { resolution }\end{array}$ & $\begin{array}{c}\text { Magnetic } \\
\text { field }\end{array}$ & $\begin{array}{c}\text { Possible active } \\
\text { media }\end{array}$ \\
\hline SDC & hadron & $<1.0 / \sqrt{E} \otimes 0.05$ & absent & $\begin{array}{c}\text { warm liquid, liq.scint. } \\
\text { fibres, high pres.gases }\end{array}$ \\
\hline $\mathbf{L}^{\star}$ & hadron & $54 \% / \sqrt{E}+2 \%$ & absent & warm liquid \\
el.magnetic & $17 \% / \sqrt{E}+1 \%$ & TMS \\
\hline EMPACT & hadron & $0.5 / \sqrt{E}+0.02$ & absent & liq.argon, SPACAL \\
\hline LHC $^{+}$ & hadron & $\sqrt{\frac{(0.65)^{2}}{\sqrt{E}}+\frac{(0.1)^{2}}{E^{2}}}$ & absent & $\mathrm{Xe} / \mathrm{CH}_{4}$ \\
\hline
\end{tabular}

$\otimes$ - term added quadratically

+ - C.W.Fabjan private communication

Table 2: Possible candidates for forward calorimeter

\begin{tabular}{|c|c|c|c|}
\hline $\begin{array}{c}\text { Calorimeter } \\
\text { type }\end{array}$ & $\begin{array}{c}\text { Maximum yearly } \\
\text { dose, Mrad }\end{array}$ & $\begin{array}{c}\text { Element, determining } \\
\text { radiation hardness }\end{array}$ & $\begin{array}{c}\text { Information } \\
\text { sourse }\end{array}$ \\
\hline $\begin{array}{c}\text { ionization, warm } \\
\text { liquids, cold } \\
\text { (noble) liquids, } \\
\text { compressed gases }\end{array}$ & $\leq 10$ & $\begin{array}{c}\text { semiconductor } \\
\text { elements of } \\
\text { electronics }\end{array}$ & $\begin{array}{c}\text { LHC } \\
\text { WORKSHOP } \\
\text { Oct.1990 } \\
\text { Aachen }\end{array}$ \\
\hline $\begin{array}{c}\text { single cristal, } \\
\text { scintillation } \\
\text { and Cerencov }\end{array}$ & $\begin{array}{c}\geq 100 \\
\text { for number } \\
\text { of materials }\end{array}$ & $\begin{array}{c}\text { single crystal, scin- } \\
\text { tillator or radiator, } \\
\text { photocathode }\end{array}$ & $/ 3 /$ \\
\hline $\begin{array}{c}\text { based on secondary } \\
\text { emission: MCP, } \\
\text { metall emitters }\end{array}$ & $?$ & $\begin{array}{c}\text { MCP, } \\
\text { emitters }\end{array}$ & $\begin{array}{c}/ 4 / \\
\text { present work }\end{array}$ \\
\hline
\end{tabular}


Table 3: Results on calculation of secondary emission flight type calorimeter

\begin{tabular}{|c|c|c|c|c|c|c|c|c|c|}
\hline$N$ & $\begin{array}{c}\mathrm{E} \\
\mathrm{GeV}\end{array}$ & $\begin{array}{l}\text { Emis. } \\
\text { prob. }\end{array}$ & $\begin{array}{c}\text { Pb-thick } \\
\text { (cm) }\end{array}$ & $\begin{array}{l}\text { Segmenta- } \\
\text { tion }\end{array}$ & $\begin{array}{c}\sigma \mathrm{E} / \mathrm{E} \\
(\%)\end{array}$ & $N_{e}$ & $\begin{array}{l}\mathrm{E} \\
(\%)\end{array}$ & $\begin{array}{c}\mathrm{S} \\
\left(\mathrm{cm}^{2}\right)\end{array}$ & $k_{\text {mult }}$ \\
\hline 1 & 2 & 3 & 4 & 5 & 6 & 7 & 8 & 9 & 10 \\
\hline 1 & 5 & 0,05 & 0,15 & & 33,5 & 1266 & 93 & $52 \times 52$ & 1 \\
\hline 2 & 5 & 0,05 & 0,3 & & 31,3 & 736 & 94 & $52 \times 52$ & 1 \\
\hline 3 & 5 & 0,05 & 0,5 & & 31,2 & 510 & 94 & $52 \times 52$ & 1 \\
\hline 4 & 5 & 0,05 & 0,8 & & 31,3 & 433 & 96 & $52 \times 52$ & 1 \\
\hline 5 & 5 & 0,05 & 1,2 & & 32,2 & 370 & 95 & $52 \times 52$ & 1 \\
\hline 6 & 5 & 0,025 & 0,15 & & 46,5 & 648 & 93 & $52 \times 52$ & 1 \\
\hline 7 & \multicolumn{3}{|c|}{ (No Poisson fluctuations) } & & 15,2 & 635 & 93 & $52 \times 52$ & 1 \\
\hline 8 & 5 & 0,025 & 0,15 & & 51,3 & 1326 & 92 & $52 \times 52$ & 1,03 \\
\hline 9 & 5 & 0,025 & 0,15 & & 98,8 & 73515 & 92 & $52 \times 52$ & 1,165 \\
\hline 10 & 5 & 0,025 & 0,15 & $\stackrel{25,25}{\rightarrow}$ & 34,5 & 638 & 92 & $52 \times 52$ & 1 \\
\hline 11 & 5 & 0,025 & 0,15 & 25,25 & 37,6 & 637 & 93 & $52 \times 52$ & 1 \\
\hline 12 & 5 & 0,025 & 0,15 & 24,26 & 29,5 & 633 & 93 & $52 \times 52$ & 1 \\
\hline 13 & 5 & 0,025 & 0,15 & $18,10,10,12$ & 24,9 & 635 & 93 & $52 \times 52$ & 1 \\
\hline 14 & 5 & 0,025 & 0,15 & $18,10,10,12$ & 29,2 & 1427 & 93 & $52 \times 52$ & 1,165 \\
\hline 15 & 5 & 0,025 & 0,15 & & 55,4 & 467 & 78 & $10 \times 10$ & 1 \\
\hline 16 & \multicolumn{4}{|c|}{ (No Poisson fluctuations) } & 15,4 & 462 & 78 & $10 \times 10$ & 1 \\
\hline 17 & 5 & 0,025 & 0,15 & \multirow{3}{*}{$18,10,10,12$} & 91,8 & 20057 & 78 & $10 \times 10$ & 1,13 \\
\hline 18 & 5 & 0,025 & 0,15 & & 31,4 & 1020 & 78 & $10 \times 10$ & 1,165 \\
\hline 19 & 5 & 0,025 & 0,3 & & 41,8 & 361 & 90 & $52 \times 52$ & 1 \\
\hline 20 & \multicolumn{3}{|c|}{ (No Poisson fluctuations) } & & 19,2 & 359 & 90 & $52 \times 52$ & 1 \\
\hline 21 & 5 & 0,025 & 0,3 & \multirow{3}{*}{ 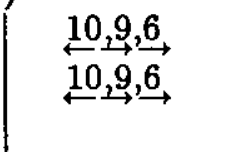 } & 32,3 & 359 & 90 & $52 \times 52$ & 1 \\
\hline 22 & 5 & 0,05 & 0,3 & & 25,0 & 717 & 90 & $52 \times 52$ & 1 \\
\hline 23 & 26 & 0,025 & 0,15 & & 57,9 & 2387 & 76 & $10 \times 10$ & 1 \\
\hline 24 & \multicolumn{3}{|c|}{ (No Poisson fluctuations) } & & 33,5 & 2382 & 76 & $10 \times 10$ & 1 \\
\hline 25 & 26 & 0,025 & 0,15 & & 128,1 & 411 & 76 & $10 \times 10$ & 0,90 \\
\hline 26 & 26 & 0,025 & 0,15 & & 76,1 & 890 & 76 & $10 \times 10$ & 0,95 \\
\hline 27 & 26 & 0,025 & 0,15 & & 84,3 & 4768 & 76 & $10 \times 10$ & 1,03 \\
\hline 28 & 26 & 0,025 & 0,15 & & 117,2 & 10140 & 76 & $10 \times 10$ & 1,06 \\
\hline 29 & 26 & 0,025 & 0,15 & & 163,1 & 29853 & 76 & $10 \times 10$ & 1,10 \\
\hline 30 & 26 & 0,025 & 0,15 & & 188,5 & 69410 & 76 & $10 \times 10$ & 1,13 \\
\hline 31 & 26 & 0,025 & 0,15 & & 201,4 & 106900 & 76 & $10 \times 10$ & 1,145 \\
\hline 32 & 26 & 0,025 & 0,15 & & 220,4 & 191339 & 76 & $10 \times 10$ & 1,165 \\
\hline 33 & 26 & 0,025 & 0,15 & $\stackrel{18}{\rightarrow}, 10,10,12$ & 35,7 & 5176 & 76 & $10 \times 10$ & 1,165 \\
\hline 34 & 26 & 0,05 & 0,15 & & 185,2 & 138091 & 76 & $10 \times 10$ & 1,13 \\
\hline
\end{tabular}

